UNIVERSIDADE DE SÃO PAULO

INSTITUTO DE GEOCIÊNCIAS

HETEROGENEIDADES EM MULTIESCALA DA FORMAÇÃO

MARIZAL (APTIANO, BACIA DO TUCANO, BA) COMO

ANÁLOGO DE RESERVATÓRIOS FLUVIAIS EM BACIAS

DISTENSIONAIS

Natalia Naches Hilbert

Orientadora: Profa. Dra. Liliane Janikian Paes de Almeida

DISSERTAÇÃO DE MESTRADO

Programa de Pós-Graduação em Geoquímica e Geotectônica

VERSÃO CORRIGIDA

SÃO PAULO

2016 


\title{
HETEROGENEIDADES EM MULTIESCALA DA FORMAÇÃO MARIZAL (APTIANO, BACIA DO TUCANO, BA) COMO ANÁLOGO DE RESERVATÓRIOS FLUVIAIS EM BACIAS DISTENSIONAIS
}

\author{
Dissertação apresentada ao Instituto \\ de Geociências da Universidade de São \\ Paulo para obtenção de título de Mestre \\ em Geologia. \\ Área de concentração: Geotectônica \\ Orientadora: Profa. Dra. Liliane \\ Janikian Paes de Almeida
}


Autorizo a reprodução e divulgação total ou parcial deste trabalho, por qualquer meio convencional ou eletrônico, para fins de estudo e pesquisa, desde que citada a fonte.

Ficha catalográfica preparada pelo Serviço de Biblioteca e Documentação do Instituto de Geociências da Universidade de São Paulo

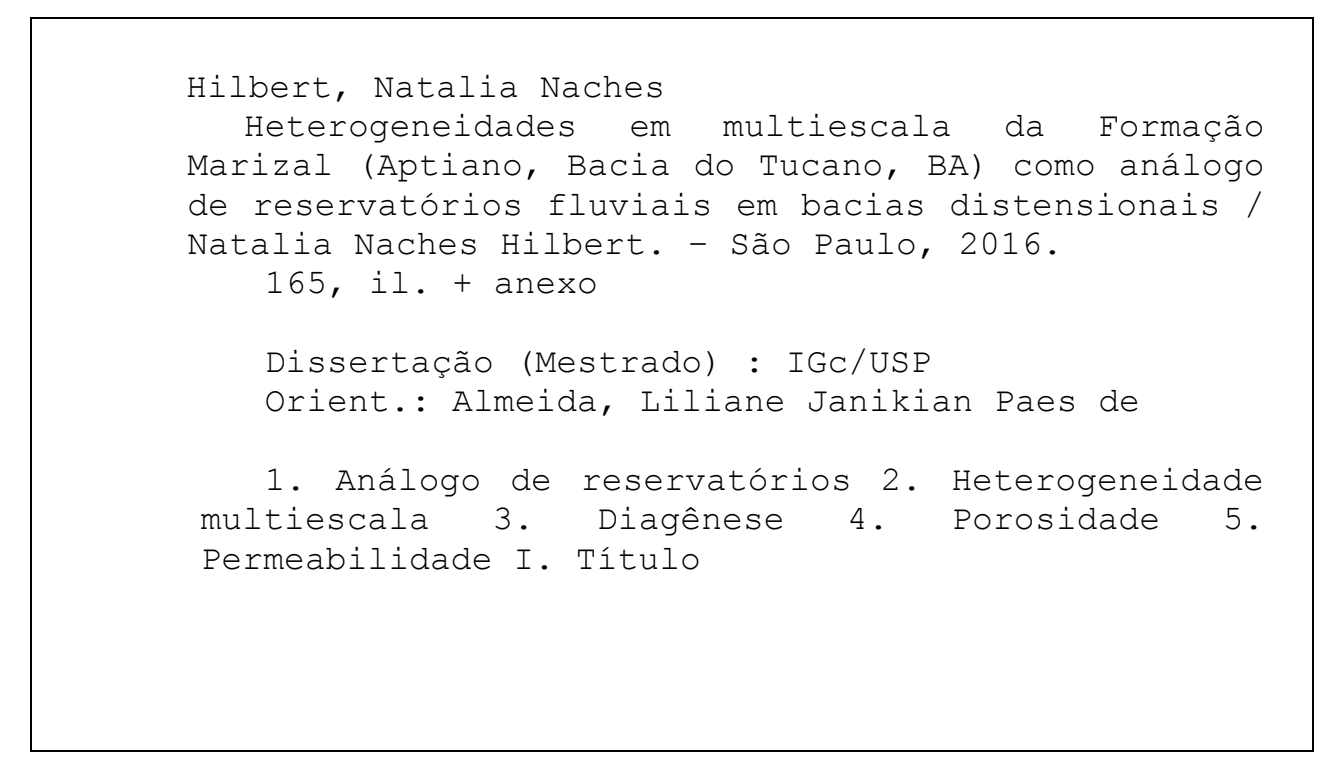


Nome: Natalia Naches Hilbert

Título: HETEROGENEIDADES EM MULTIESCALA DA FORMAÇÃO MARIZAL (APTIANO, BACIA DO TUCANO, BA) COMO ANÁLOGO DE RESERVATÓRIOS FLUVIAIS EM BACIAS DISTENSIONAIS

Dissertação apresentada ao Instituto de Geociências da Universidade de São Paulo para obtenção de título de Mestre em Geologia.

Área de concentração:

Geotectônica

Orientadora: Profa. Dra. Liliane Janikian Paes de Almeida

Aprovado em:

Prof. Dr.: Instituição:

Julgamento: Assinatura:

Prof. Dr.: Instituição:

Julgamento: Assinatura:

Prof. Dr.: Instituição:

Julgamento: Assinatura: 
A Eloisa, Sofia e aos Volneis 


\section{AGRADECIMENTOS}

Primeiramente, meu agradecimento a Profa. Liliane pela oportunidade e confiança em desenvolver projeto tão grandioso que eu mesma não acreditaria ser capaz de desenvolver, seu entusiasmo ao longo das realizações do trabalho foram importantes. Agradecimento especial ao Prof. Carlos Guedes por novamente confiar em meu trabalho para uma nova jornada como sua "orientanda" no projeto de mestrado, o seu incentivo foi fundamental para meu ingresso no mestrado.

Gostaria de agradecer a todos do Grupo Mocó pelas conversas sempre bem humoradas e prontidão para ajudar no que fosse preciso. Em especial, agradeço o Prof. Renato pelo entusiasmo em suas explicações sobre sistemas fluviais e o Prof. Bernardo por sua gentileza em compartilhar o seu conhecimento e os seus dados sobre a Formação Marizal, muito não teria sido obtido sem esse conhecimento prévio. Agradeço a Simone Carrera pela coleta das amostras iniciais de petrofísica e pelos fotomosaicos cedidos. Ao André (Kodornna) pela revisão do abstract. Por fim, um agradecimento mais do que especial para os que ajudaram nos longos trabalhos de campo, a Maria Paula, Cristiano, Heitor, Bia e Ramon.

A Dário Cruz pela grande ajuda na calibração dos dados petrofísicos, sem seus conhecimentos essa importante tarefa não teria sido cumprida, e ainda pela disposição e entusiasmo em ajudar nas várias dúvidas que surgiram.

A André Souza da Schlumberger pela plugagem das amostras de rocha, pelos inúmeros favores e pela recepção no Rio de Janeiro. Ao Prof. Rodrigo Bagueira e Prof. José Afrânio Brenelli da UFF pela realização das análises petrofísicas.

Ao Prof. Gelson Fambrini pelo apoio e ensinamentos na descrição do testemunho e pelo trabalho de campo e ao Prof. Virgínio Neumann por disponibilizar o testemunho da Formação Marizal para descrição e coleta de amostras.

Ao Prof. Luis Fernando De Ros pelas ajudas online sobre diagênese. A Denise Stolnik por me receber em sua casa com muito carinho durante o tempo que cursei a disciplina de diagênese.

A Jordana e Isaac pela sempre pronta disponibilidade em ajudar e auxiliar, os 4 meses que passei no LabPetro não teriam sido o mesmo sem nossas conversas. E a todos os funcionários que contribuíram para o desenvolvimento dessa pesquisa.

A Fapesp pelo apoio financeiro sob a forma do processo 2013/01825-3 (Auxílio a Pesquisa) e ao PRH -241 pelo auxilio através de bolsa.

Por fim, mas com muito carinho, aos meus amigos pelo apoio, conversas e desabafos. Vocês com certeza foram a força e animação para que essa etapa fosse concluída.

A minha família, a quem acredito que não seja necessário expressar meus sentimentos, essa obra é a nossa criação. 


\section{RESUMO}

O presente trabalho realizou a caracterização faciológica, petrofísica e diagenética de análogos de reservatório em afloramentos de depósitos fluviais e eólicos da Formação Marizal, Aptiano, Bacia do Tucano (BA), buscando agregar novos conhecimentos em relação a heterogeneidades em multiescala para o entendimento dos processos que controlam os parâmetros petrofísicos e diagenéticos.

As propriedades petrofísicas, porosidade e permeabilidade, dos arenitos da Formação Marizal são controladas pela proveniência e diagênese, além da posição dos depósitos fluviais (axial e transversal) dentro da bacia sedimentar. Para os arenitos que possuem proveniência da borda da bacia, ocorrem maiores quantidades de fragmentos líticos. Esses fragmentos, quando sujeitos a diagênese, sofrem processos de compactação mecânica que obstruem poros e gargantas de poro, levando a menores valores de porosidade e permeabilidade em relação ao depósitos de sistema fluvial axial do Membro Banzaê. Os depósitos fluviais axiais possuem composição de arenito arcoseano não tiveram obstrução de poros.

Os arenitos dos depósitos fluviais transversais do Membro Banzaê apresentam como constituintes diagenéticos franja de interestratificados I/S e caulinita mesodiagenéticas; enquanto que os depósitos axiais apresentam sobrecrescimento de k-feldspato, calcita e dolomita eodiagenéticas, além de interestratificados de I/S e caulinita mesodiagenéticas.

Para os arenitos do Membro Cícero Dantas também se observa sobrecrescimento de kfeldspato, calcita e dolomita eodiagenéticas, além de interestratificados de I/S e caulinita mesodiagenéticas.

Os constituintes telodiagenéticos para os dois membros são a ferruginização e a argila infiltrada, sendo que para o Membro Cícero Dantas esses processos foram mais significativos, tendo influência negativas sobre os valores de permeabilidade, onde se observa que os depósitos de barra dessa unidade apresentam valores similares aos mesmos elementos arquiteturais dos depósitos transversais do Membro Banzaê.

Por fim, os constituintes diagenéticos, como sobrecrescimento de k-feldspato e interestratificados I/S, indicam condições de climas semi-áridos para a Formação Marizal durante sua deposição. 


\section{ABSTRACT}

This study presents facies, petrophysical and diagenetic characterization of outcrop reservoir analogues of fluvial and aeolian deposits from the Marizal Formation, Tucano Basin, Northeastern Brazil. The study brings new knowledge to the study of multiscale heterogeneities and processes controlling petrophysical and diagenetic parameters.

The petrophysical parameters, porosity and permeability of the Marizal Formation sandstones are controlled by provenance, diagenesis, as well as the position of the deposits (axial and transverse) within the sedimentary basin. The sandstones with source areas at the basin border have large amounts of lithic fragments. When lithic fragments are subjected to diagenesis mechanical compaction process obstruct pores and pore throats, leading to lower values of porosity and permeability relative to the axial fluvial systems deposits from the Banzaê Member. Moreover, the axial deposits have arkosic composition, without the lithic fragments that obstruct pores.

The sandstones from the Banzaê Member transverse fluvial deposits present illite-smectite mixed layers and kaolinite as mesodiagenetic constituents; while the axial deposits have eodiagenetic K-feldspar overgrowths, calcite and dolomite, besides mesodiagenetic illite-smectite mixed layers and kaolinite.

The Cícero Dantas Member sandstones also have eodiagenetic K-feldspar overgrowths, calcite and dolomite, and mesodiagenetic illite-smectite mixed layers and kaolinite.

The telodiagenetic constituents for the two members are ferruginization and infiltrated clay. These processes were more important, with influence on the permeability, in the Cícero Dantas Member, whose bar deposits have values similar to the bar deposits from the transverse deposits from the Banzaê Member.

Finally, the diagenetic constituents, such as k-feldspar overgrowth and illite-smectite mixed layers, indicate semi-arid climate for the Marizal Formation during deposition. 


\section{LISTA DE FIGURAS}

Figura 1. Mapa com localização geográfica dos afloramentos com principais cidades (esquerda) e mapa geológico com enfoque na distribuição da unidade Aptiana (extraído de Freitas, 2014) e também localização dos afloramentos em contexto geológico.

Figura 2. Bacia do Recôncavo-Tucano-Jatobá e embasamento adjacentes pertencentes as Províncias São Francisco e Borborema (Modificado de Santos \& Reis, 2011)........................ 4

Figura 3. Carta estratigráfica da Bacia do Tucano (Extraído de Santos et al., 2010)................7

Figura 4. Coluna estratigráfica da Formação Marizal (Extraído de Santos et al., 2010). ........ 10 Figura 5. Mapa geológico do sistema de Rifte Recôncavo-Tucano-Jatobá, atentar para a divisão da Formação Marizal nos Membros Banzaê e Cícero Dantas e sua distribuição em área. Falhas maiores: A - Falha de Ibimirim; Falha de São Saité; C - Falha de Caritá; D - Falha de Umburana; E - Falhas de Massacará, Barrigató, Aribicé e Fazenda Murici; F - Falha de Adustina; Falha de Salvador. Extraído de Freitas (2014)...................................................... 12 Figura 6. Empacotamento cúbico e romboédrico, notar que diferentes tipos de empacotamento terão diferentes valores de porosidade (Extraído de Selley, 1998). 15

Figura 7. Gráfico apresenta porosidade x permeabilidade em relação a tamanho de grão e seleção granulométrica (Extraído de Selley, 1998).

Figura 8A. Desenho esquemático apresenta constituintes comuns da diagênese e a sua evolução na preservação da porosidade (A) e na redução da porosidade (B) (Extraído de Morad et al., 2010) 18

Figura 9. Classificação de estilos de canais e suas variáveis associadas (Extraído de Schumm, 1985). 22

Figura 10. Evolução dos estilos de canais. Gráfico apresenta declividade, média do tamanho de sedimento transportado e descarga de água como controles de sinuosidade e grau de entrelaçamento (Extraído de Bridge, 2006).

Figura 11. Esquema dos elementos morfológicos do rio entrelaçado com destaque as depósitos de fundo de canal, barras unitárias e topo de barra (Sambrook et al., 2006 modificado de Walker \& Cant, 1978).

Figura 12. Estrutura das barras laterais, longitudinais e transversais em rio entrelaçado (Extraído de Boggs, 1995).

Figura 13. Distribuição esquemática das formas de leito e granulometria. O limite entre areia grossa e fina é gradual (Extraído de Bridge, 2006).

Figura 14. Diagrama tridimensional com os principais estilos deposicionais de barra longitudinal no Rio Jamuna, Bangladesh (Extraído de Best et al., 2003). 28 
Figura 15. Fatores envolvidos na formação dos estratos cruzados (Extraído de Reesink \& Bridge, 2009).

Figura 16. Formação de estratos cruzados por migração de formas de leito menor do que a hospedeira (A) e maior do que a hospedeira (B) (Extraído de Reesink \& Bridge, 2007). 30 Figura 17. Estratos cruzados em diferentes taxas de sedimentação. As flechas apontam diminuição na granulometria (Extraído de Reesink \& Bridge, 2009).

Figura 18. Distribuição das fácies eólicas em uma duna barcana em larga e pequena escala (extraído de Mountney, 2006).

Figura 19. Diagrama esquemático apresenta em 3D estruturas de menor escala que compõem as dunas (Extraído de Mountney, 2006).

Figura 20. Classificação das estratificações wind-ripple em relação ao ângulo de cavalgamento e presença de laminação cruzada. (Extraído de Mountney, 2006).

Figura 21. Tipos de estratificações e variações na face de avalanche (Extraído de Kocurek, 1996)

Figura 22. Estruturas e feições características de depósitos de interduna em relação a cada condição deposicional. (Extraído de Kocurek, 1981)...

Figura 23. Sequência de estruturas que preenchem uma interduna em transição úmida-seca (Extraído de Kocurek, 1981).

Figura 24. Esquema sumarizando os tipos de interações fluvio-eólico (Extraído de Al-Masrahy \& Mountney, 2015).

Figura 25. Estilos de estratos cruzados em sistema eólico, setas apresentam direção da permeabilidade, quanto mais setas maior o potencial de fluxo. (A) Fluxo de grãos, (B) Windripple, (C) Intercalação entre fluxo de grãos e wind-ripple e (D) interdigitação entre fluxo de grãos e wind-ripple (Extraído de Chandler et al., 1989).

Figura 26. Variabilidade lateral e vertical em estratificações e em elementos arquiteturais em sistema eólico. Setas apresentam direção do fluxo, linhas pontilhadas apresentam descontinuidades devido a mudanças no tipo de estratificação (Extraído de Lindquist, 1989).

Figura 27. Esquema dos controles da diagênese clástica. Extraído de De Ros (1996).

Figura 28. A, Agregados vermiculares de cristais euhedrais de caulinita autigênica que preenche poro; fotomicrografia MEV (Extraído de Khidir \& Catuneanu (2010); B, Esmectita autigênica como franja; fotomicrografia MEV (Extraído de Khidir \& Catuneanu (2010); C, Fotomicrografia óptica apresenta cutículas irregulares de argila mecanicamente infiltrada; polarizadores cruzados (Extraído de De Ros \& Scherer, 2012); D, Arenito cimentado por calcita poiquilotópica pós-compactacional; fotomicrografia óptica com polarizadores cruzados 
(Extraído de De Ros \& Scherer, 2012); E, Sobrecrescimento autigênico de k-feldspato (Kfo) apresenta faces euhedrais cobertas por argila infiltrada (Ic) e cimento calcítico (Ca); fotomicrografia óptica com polarizadores cruzados (Extraído de Maraschin et al., 2004); F, Sobrecrescimento sintaxial de quartzo; fotomicrografia (Extraído de Reed et al., 2005).

Figura 29. Mecanismos e locais de infiltração de argila. A, o acúmulo de argila se dá na zona vadosa pela evaporação da água infiltrada. B, acumulação de argila se deve a perda de energia da água infiltrada quando atinge o lençol freático. C, acúmulo de argila se desenvolve sobre superfícies impermeáveis devido ao acumulo de água sobre essa superfície. D, associação entre acumulação de argila e fontes com influente infiltração como altos do embasamento e canais aluviais (Extraído de Moraes \& De Ros, 1992). 46

Figura 30. Ilustração esquemática das 3 direções (V, H1 e H2) que foram marcadas em cada amostra. Modificado de Meyer \& Krause (2006).

Figura 31. Representação esquemática do método de Gazzi-Dickinson que minimiza a dependência da composição da rocha em relação a granulometria (Extraído de Zuffa, 1985).

Figura 32. Gráfico de Houseknecht (1987) apresenta campos em que a perda de porosidade original (considerada pelo autor como de 40\%) se deu pela compactação mecânica e/ou química ou pela cimentação.

Figura 33. Método de segmentação dos componentes HSI. Na imagem à esquerda distingue-se o espaço poroso (p) impregnado com resina azul do arcabouço (np). Através dos ajustes dos componentes HSI obtém-se a segregação dos poros (imagem à direita, poro em vermelho) do arcabouço. Posteriormente, caso necessário, realiza-se correção manual, para que haja total coincidência da imagem com os poros da amostra. Por fim, obtém-se o valor porcentual de porosidade discriminado na imagem.

Figura 34. Gráfico apresenta correlação entre dados obtidos pelo TinyPerm e dados obtidos em laboratório pelo método a gás.

Figura 35. Fotomosaico com detalhes das estruturas encontradas (Extraído de Freitas, 2014).

Localização das amostras estão representadas através de seus números. 61

Figura 36. Seções colunares (S1 - S5) apresentam disposições das fácies, seções localizadas na figura abaixo (Extraído de Freitas, 2014).

Figura 37. Localização de todas as amostras retiradas para análise quanto a porosidade e a permeabilidade através do método a gás.

Figura 38. Boxplot apresenta a variação da permeabilidade e da porosidade em relação a direção $(A, B)$, granulometria $(C, D)$, seleção granulométrica $(E, F)$ e fácies $(G, H)$. 
Figura 39. Boxplot apresenta a variação da permeabilidade e da porosidade em relação a fácies e direção (A e B) e em relação a fácies e seleção granulométrica (C e D).

Figura 40. Gráficos de dispersão entre porosidade e permeabilidade, geral (A), em relação a direção (B) e as fácies (C).

Figura 41. Fotomosaico interpretado com associação de fácies e superfícies limitantes. Números apresentam a localização dos dados de permeabilidade obtidos através do permeâmetro de campo. Aga - arenito grosso com estratificação cruzada acanalada, Aca arenito cascalhento com estratificação cruzada acanalada, Ama - arenito médio com estratificação cruzada acanalada e Ca - conglomerado com estratificação cruzada acanalada.

Figura 42. Fotomosaico do afloramento MR-03 com seções colunares levantadas.

Figura 43. Fotos das fácies sedimentares descritas no afloramento MR-03 (Membro Banzaê transversal). A, na porção mediana da foto ocorre arenito grosso com seixos nos foresets (Aga) e acima é truncado pelo arenito médio com estratificação cruzada acanalada (Ama). B, Detalhe da fácies Aga da foto A. C, fácies Aca, notar blocos e seixos nos foresets da acanalada........ 70 Figura 44. Boxplot com dados de permeabilidade em relação as fácies que representam a associação de fácies de barras arenosas subaquáticas.

Figura 45. Fotomosaico com interpretação das associações de fácies e superfícies limitantes. Números apresentam a localização dos dados de permeabilidade obtidos através do permeâmetro de campo. Aftg - arenito fino com estratificação cruzada tangencial, Amtg arenito médio a grosso com estratificação cruzada tangencial e Actg - arenito conglomerático com estratificação cruzada tangencial. 73

Figura 46. Fotomosaico do afloramento MR-05 com seções colunares levantadas 74 Figura 47. Fotos das fácies e superfícies presentes no afloramento MR-05. A, arenito fino avermelhado com estratificação cruzada tangencial (Aftg). B, superfície cascalhenta, com seixos e blocos que separa as fácies Aftg e Amtg. C, arenito médio com estratificação cruzada tangencial e poucos seixos esparsos.

Figura 48. Boxplot com os dados de permeabilidade em relação as fácies presentes no afloramento 76

Figura 49. Fotomosaico interpretado para associação de fácies e superfície limitante. Ci conglomerados de seixos imbricados, Amt - arenito médio com estratificação cruzada tabular e Ama - arenito médio a grosso com estratificação cruzada acanalada. 78

Figura 50. Fotomosaico com seções colunares levantadas. 79

Figura 51. Fotos das fácies do afloramento NAT-01 (Membro Banzaê - Transversal). A, Fácies Amt, arenito médio com estratificação cruzada tabular. B, Fácies Ama, arenito médio com 
estratificação cruzada acanalada. C, Detalhe da fácies Ama, conglomerados de seixos na base das acanaladas

Figura 52. Fotomosaico interpretado quanto as associações de fácies e superfícies limitantes para o afloramento SI-34. Números apresentam posição da onde se retirou amostras (A, B, C, D, E, F, G, H e I) e coleta de dados de permeabilidade através do permeâmetro de campo (147). Afp - arenito fino com estratificação plano-paralela e Afa - arenito fino com estratificação cruzada acanalada.

Figura 53. Fotomosaico do afloramento SI-34 com seções colunares levantadas. 84

Figura 54. Fotos das fácies sedimentares presentes no afloramento SI-34. A, Detalhe da fácies Afp (arenito com estratificação plano-paralela), notar níveis mais mais escuros (micáceos) intercalados com níveis mais claros, definindo a estratificação. B, Concreção na fácies Afp. C, Detalhe da fácies Afa (arenito com estratificação cruzada acanalada), notar estratos cruzados bem marcados. D, Concreção presenta na fácies Afa. 85

Figura 55. Boxplot apresenta a relação da porosidade e permeabilidade com a direção (A e B), fácies $(C, D)$ e direção em relação a fácies $(E$ e F). 86

Figura 56. Gráfico de dispersão apresenta relação direta entre permeabilidade e porosidade (A), dividido pela direção (B) e fácies (C)

Figura 57. Fotomosaico geral do afloramento NAT-02 e seções colunares levantadas. 90 Figura 58. Fotomosaico do Perfil I com associação de fácies e superfícies limitantes interpretadas. Números apresentam localização da amostragem (1, 3 e 4) e coleta de dados de permeabilidade através do permeâmetro de campo $(1-6 ; 27-62)$. 91

Figura 59. Fotomosaico do Perfil II com associação de fácies e superfícies limitantes interpretadas. Números apresentam localização da amostragem $(7,8,9,10,11,12,13,14,15$ e 17) e coleta de dados de permeabilidade através do permeâmetro de campo (7-17; 63-83)....92 Figura 60. Fotomosaico do Perfil III com associação de fácies e superfícies limitantes interpretadas. Números apresentam localização da amostragem $(18,22,23$ e 24) e coleta de dados de permeabilidade através do permeâmetro de campo (18-25; 85-96). Aftg - arenito fino com estratificacão cruzada tangencial, Afs - arenito fino com cruzada sigmoidal, Ac - arenito fino com estratificação convoluta e Alc - arenito fino com laminação cruzada cavalgante. ...93 Figura 61. Fotos das fácies sedimentares do afloramento NAT-02, localizado próximo a cidade de Sátiro Dias (BA). A, Fácies Afs, sigmoíde com aplainamento no topo e na base. B, C Fácies Ac, arenito convoluto. D, Fácies Alc notar laminação cruzada cavalgante subcrítica. 94 Figura 62. Boxplot apresentam a variação da permeabilidade e da porosidade em relação a direção (A e B), fácies (C e D) e elemento arquitetural (E e F) e também variação da permeabilidade em relação a direção em cada fácies $(\mathrm{G}$ e F). 
Figura 63. Gráficos de dispersão entre os dados de porosidade e permeabilidade geral (A), em relação a direção (B), fácies (C) e elemento arquitetural (D).............................................. 96

Figura 64. Fotomosaico geral do afloramento NAT-03 e seções colunares levantadas............99 Figura 65. Fotomosaico do Perfil I com associação de fácies e superfícies limitantes interpretadas. Números apresentam localização da amostragem $(1,2,4,5,7,8,9)$ e coleta de dados de permeabilidade através do permeâmetro de campo (1-9; 30-56).

Figura 66. Fotomosaico do Perfil II com associação de fácies e superfícies limitantes interpretadas. Números apresentam localização da amostragem (10, 13, 17, 19, 25 e 29) e coleta de dados de permeabilidade através do permeâmetro de campo (10-25 e 29; 57-85). H - arenito fino heterolítico, Alc - arenito fino com laminação cruzada cavalgante, Afp - arenito fino com estratificação cruzada de baixo ângulo e Aftg - arenito fino com estratificação cruzada tangencial. 101

Figura 67. Fotomosaico do Perfil III com associação de fácies e superfícies limitantes interpretadas. Números apresentam coleta de dados de permeabilidade através do permeâmetro de campo (26-28; 86-96). 102

Figura 68. Fotos sedimentares das fácies sedimentares do afloramento NAT-03, localizado na entrada da cidade de Sátiro Dias (BA). A, Fácies H, arenito fino siltoso heterolítico. B, Fácies Alc arenito fino com laminação cruzada cavalgante subcrítica C, Fácies Afp, arenito fino com estratificação de baixo ângulo. D, Fácies Aftg, arenito fino com estratificação cruzada tangencial. 103

Figura 69. Boxplot apresenta dados de porosidade e permeabilidade e sua variação em relação a direção (A e B), fácies (C e D) e elemento arquitetural (E e F). 104

Figura 70. Boxplot apresenta valores de porosidade permeabilidade em relação a direção para cada fácies (A e B) e as fácies em relação ao elemento arquitetural (C e D). 105 Figura 71. Gráficos de dispersão entre dados de porosidade e permeabilidade geral (A), em relação a direção (B), fácies (C) e elemento arquitetural (D).............................................. 106

Figura 72. Fotomosaico geral do afloramento NAT-04 e seções colunares levantadas. 109 Figura 73. Fotomosaico do Perfil I com associação de fácies e superfícies limitantes interpretadas. Números apresentam localização da amostragem $(1,4,6,9,10,11,13$ e 17) e coleta de dados de permeabilidade através do permeâmetro de campo (1-18; 43-45). At - arenito fino a grosso com estratificação cruzada tabular, Atg - arenito fino a médio com estratificação cruzada tangencial na base, Ap - arenito fino a grosso com cruzada tabular de baixo ângulo, Alc - arenito fino com laminação cruzada cavalgante, Slc - siltito com laminação cruzada cavalgante e S1 - siltito laminado. 110 
Figura 74. Fotomosaico do Perfil II com associação de fácies e superfícies limitantes interpretadas. Números apresentam localização da amostragem (47, 49, 51, 52, 54, 55, 56, 57 , 59 e 60) e coleta de dados de permeabilidade através do permeâmetro de campo (19-42; 46-62; 63-80).

Figura 75. Fotomosaico do Perfil III com associação de fácies e superfícies limitantes interpretadas. Números apresentam coleta de dados de permeabilidade através do permeâmetro de campo (81-131).

Figura 76. Fotomosaico do Perfil IV com associação de fácies e superfícies limitantes interpretadas. Números apresentam coleta de dados de permeabilidade através do permeâmetro de campo (132-159).

Figura 77. Fotos das fácies sedimentares do afloramento NAT-04, localizado próximo ao vilarejo de Melancia. A, arenito fino com estratificação cruzada tabular. B, arenito fino com estratificação cruzada tangencial na base. C, siltito com laminação cruzada cavalgante. D. arenito fino com laminação cruzada cavalgante.

Figura 78. Boxplot apresenta dados de porosidade e permeabilidade e sua variação em relação a direção (A e B), granulometria (C e D), fácies (E e F) e elemento arquitetural $(\mathrm{G} \mathrm{e} \mathrm{H) \ldots ...115}$ Figura 79. Boxplot apresenta valores de porosidade permeabilidade em relação a direção para cada fácies (A e B).

Figura 80. Gráficos de dispersão entre dados de porosidade e permeabilidade geral (A), em relação a direção (B), fácies (C) e elemento arquitetural (D).

Figura 81. Localização das amostras do testemunho SM em seção construída por Varejão (2015), analisadas quanto a proveniência e diagênese (Modificado de Varejão, 2016). 118

Figura 82. Composição detrítica dos arenitos sistema fluvial transversal, classificação de acordo com Folk (1974).

Figura 83. A, Fragmentos lítico dissolvido e compactado, ocupando espaço intergranular. B, Compactação mecânica evidenciado pela biotita. C, Argila autigênica illita-esmectita (I/S) substituindo grão feldspático em suas clivagens. D, Franja de illita-esmectita (I/S) recobrindo grãos detríticos, notar continuidade e cristalinidade. A,B, fotomicrografias polarizador descruzado; C,D, fotomicrografias com polarizador cruzado. 121

Figura 84. Diagrama apresenta proporção de redução de porosidade intergranular por compactação e cimentação para os arenitos fluviais transversais (cf. Houseknecht, 1987)...122 Figura 85. Composição detrítica dos arenitos sistema fluvial axial, classificação de acordo com Folk (1974).

Figura 86. A, Biotita com sinais de compactação mecânica. B, Sobrecrescimento de k-feldspato (Kfo) recoberto por franja de illita-esmectita (I/S). C, Sobrecrescimento de k-feldspato epitaxial 
apresenta faces euhedrais, notar descontinuidade óptica entre grão e sobrecrescimento, sobrecrescimento encontra-se envolvido por calcita poiquilotópica. D, Esqueleto de feldspato alterado e dissolvido e sobrecrescimento de k-feldspato preservado devido a sua pureza. A,D, fotomicrografias com polarizador descruzado; B, C, fotomicrografias com polarizador cruzado.

Figura 87. A, Grão de k-feldspato dissolvido (Kfog) e sobrecrescimento de k-feldspato preservado (Kfo). B,C, Caulinita (K) intergranular recobrindo franja de illita-esmectita (I/S), notar agregados vermiculares e booklets. A, MEV micrografia; B, fotomicrografia com polarizador descruzado; $\mathrm{C}$, fotomicrografia com polarizador cruzado.

Figura 88. A, B, Cutículas de óxido de ferro $(\mathrm{Fe})$, notar sua ausência no contato entre os grãos. C, Nódulo carbonático com dolomita zonada com Fe no centro e calcita em rosa (carbonato tingido com Alizarina). D, Cristal de dolomita romboédrico zonado com $\mathrm{Fe}$ no espaço intergranular. A, MEV micrografia; B, C fotomicrografia polarizador descruzado; D, fotomicrografia com polarizador cruzado.

Figura 89. A, Dolomita deslocante causando expansão de biotita. B, Franja de illita-esmectita (I/S) recobrindo grãos detríticos, notar continuidade e cristalinidade. C, Argila autigênica illitaesmectita (I/S) substituindo grão feldspático em suas clivagens. A, fotomicrografia polarizador descruzado; B, C, fotomicrografia polarizador cruzado.

Figura 90. A,B, Cutículas de argila infiltrada (Ai) recobrindo grãos detríticos e sobrecrescimento de k-feldspato (Kfo), notar ausência da argila nos contatos entre os grãos e orientação paralela ao grão. Argila infiltrada conectando dois grãos (P), feição típica de zona vadosa. C, Argila infiltrada (Ai) criptocristalina e orientação paralela ao grão. D, Argila infiltrada (Ai) recobrindo franja de illita-esmectita (I/S). A, fotomicrografia polarizador descruzado; B, fotomicrografia polarizador cruzado; C,D, MEV micrografia.

Figura 91. Diagrama apresenta proporção de redução de porosidade intergranular por compactação e cimentação. (cf. Houseknecht, 1987).

Figura 92. Composição detritíca dos arenitos do Membro Cícero Dantas. Diagrama de Folk (1974). Em vermelho estão apresentados os arenitos do testemunho. 131

Figura 93. A,B Microclínio com sobrecrescimento de k-feldspato autigênico (Kfo). C, Feldspato dissolvido com sobrecrescimento (Kfo) preservado e recoberto por argila infiltrada (Ai). D, Caulinita em livrinhos $(\mathrm{Kl})$ e vermicular $(\mathrm{Kv})$. A,B,D, fotomicrografias com polarizador cruzado. C, fotomicrografias com polarizador descruzado.

Figura 94. A,B, Caulinita vermicular e em livrinhos $(\mathrm{K})$ preenchendo poro intergranular, observar ferruginização $(\mathrm{Fe})$. C, D, Calcita $(\mathrm{Ca})$ substituindo sobrecrescimento k-feldspático 
(Kfo) e microclínio dissolvido (Dis). Calcita está tingida descruzado. B,C,D, fotomicrografias com polarizador cruzado.

Figura 95. A, Esqueleto de cristais romboédricos de dolomita zonada com Fe deslocantes (D), expandindo biotita (Bt). C, Feldspato substituído por illita-esmectita em suas clivagens e margens. C, Cutículas de argila infiltrada (Ai) recobrindo sobrecrescimento de k-feldspato (Kfo). D, Cutículas de argila infiltrada de zona vadosa, notar pontes entre os grãos. A,D, fotomicrografias com polarizador descruzado. B,C fotomicrografias com polarizador cruzado.

Figura 96. Diagrama apresenta proporção de redução de porosidade intergranular por compactação e cimentação. (cf. Houseknecht, 1987). Os dados referentes às amostras do testemunho encontram-se em vermelho.

Figura 97. Gráficos boxplot apresentam valores de permeabilidade (A) e porosidade (B) em relação aos membros de compõem a Formação Marizal, Membro Banzaê e Cícero Dantas. Boxplot com os valores de permeabilidade (C) e porosidade (D) em relação as fácies que compõem os membros Banzaê e Cícero Dantas. 140

Figura 98. Gráficos boxplot apresentam valores de permeabilidade (A) e porosidade (B) em relação aos Membro Banzaê Axial e Transversal e Membro Cícero Dantas. 141 Figura 99. Gráficos boxplot apresentam valores de permeabilidade (A) e porosidade (B) dos elementos arquiteturais em relação aos membros Banzaê e Cícero Dantas e gráfico boxplot com valores de permeabilidade (C) em relação ao elemento arquitetural divididos em membros.

Figura 100. Gráficos boxplot apresentam valores de permeabilidade (A) de fácies em relação a direção H (horizontal) e V (vertical) (A) e de elementos arquiteturais em relação a direção (B).

Figura 101. Gráficos boxplot apresentam valores de porosidade (A) de fácies em relação a direção H (horizontal) e V (vertical) (A) e de elementos arquiteturais em relação a direção (B).

Figura 102. Gráfico de dispersão entre permeabilidade e porosidade sem discriminantes (A), discriminados por direção (B), fácies (C) e elemento arquitetural (D). 145 Figura 103. Sequência paragenética dos constituintes e processos que ocorrem na evolução dos arenitos da Formação Marizal, divididos em Membro Banzaê e Cícero Dantas. Comprimentos das retas são proporcionais ao grau de ocorrência.

Figura 104. Mapa do rifte RTJ, com destaque para as sub-bacias Tucano, Sul, Central e Norte, localização dos afloramentos estudados, vetor médio da paleocorrente e gráficos QFL. As cores roxas nos gráficos QFL representam amostras do Membro Cícero Dantas, enquanto as azuis os 
do Membro Banzaê. Notar que vetores médios de paleocorrente transversais apresentam maior quantidade de fragmentos líticos e paleocorrentes axiais não apresentam essa distinção. Dados de paleocorrente e mapa foram compilados de Freitas (2014) 153 Figura 105. Gráficos boxplot apresentam valores de porosidade e permeabilidade para os Membro Banzaê axial e transversal (A e B) e paras os mesmos membros em relação a fácies (C e D). Notar que mesma fácies presentes nos dois membros, fácies Aa, no membro transversal os valores de permeabilidade e porosidade são menores. 154

\section{LISTA DE TABELAS}

Tabela 1. Escala das formas fluviais e conjunto de estratos associados (Extraído de Bridge, 2006). 


\section{SUMÁRIO}

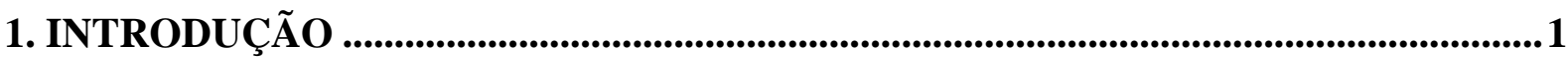

2. LEVANTAMENTO BIBLIOGRÁFICO .......................................................................3

2.1 Geologia Regional e Local................................................................................................3

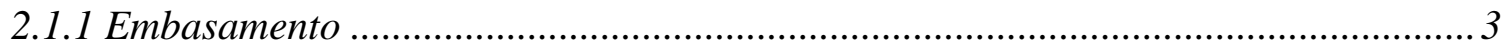

2.1.2 Preenchimento das sub-bacias Tucano central e Sul ............................................. 5

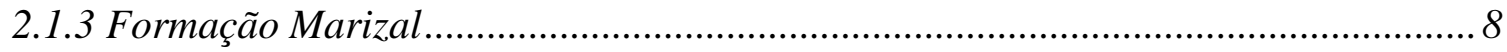

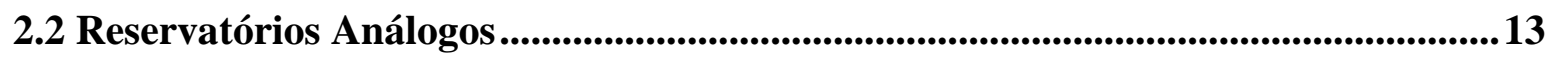

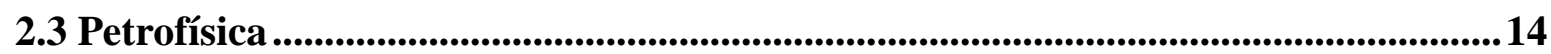

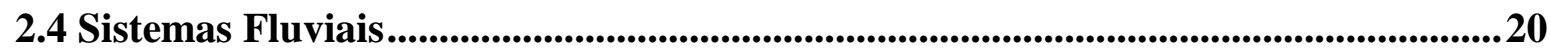

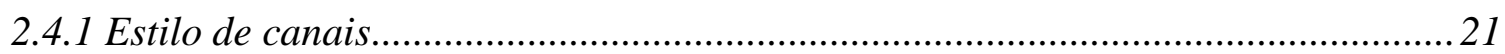

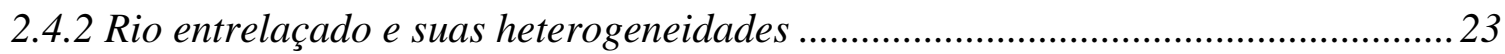

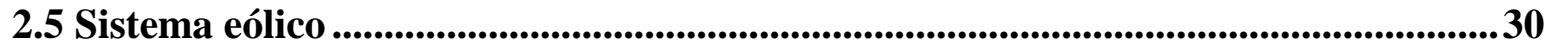

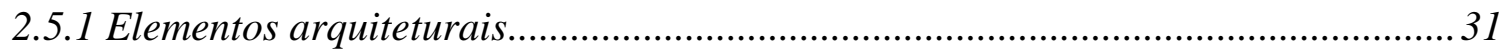

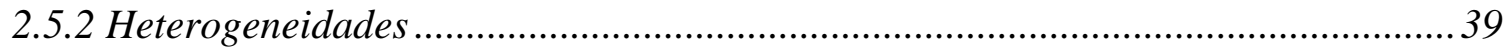

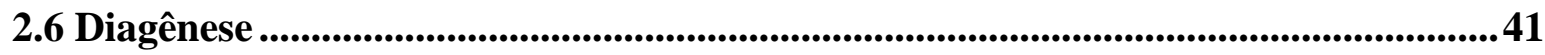

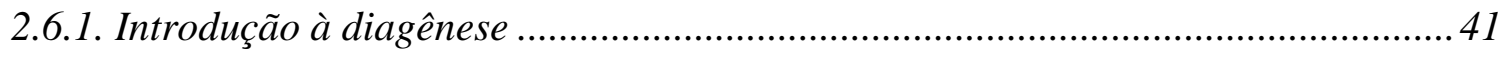

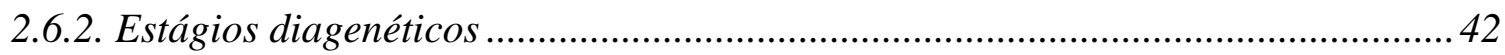

2.6.3. Fatores que controlam a diagênese ….............................................................. 42

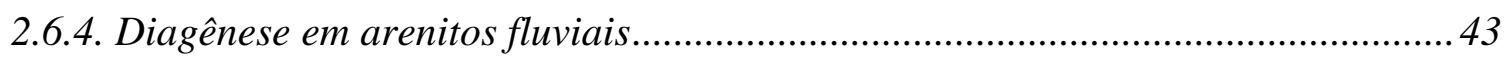

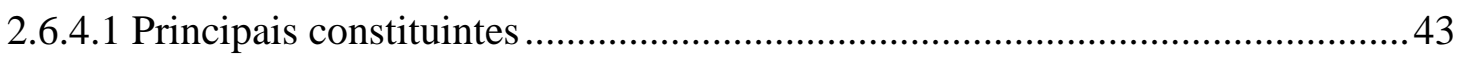

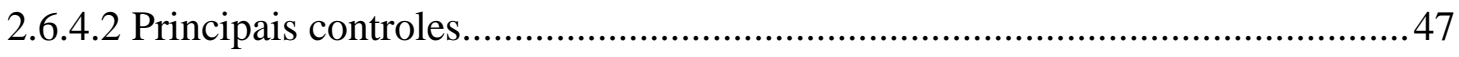

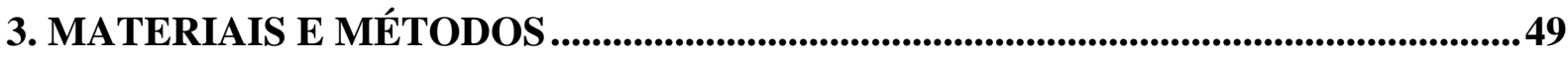

3.1 Análise de fácies sedimentares e elementos arquiteturais ....................................49

3.2 Coleta de amostras .........................................................................................................50

3.3 Preparação de amostras ...........................................................................................50

3.4 Preparação e confecção de lâminas petrográficas ..................................................51

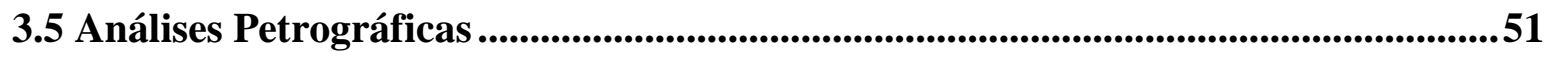


3.6 Microscopia eletrônica de varredura ............................................................................ 53

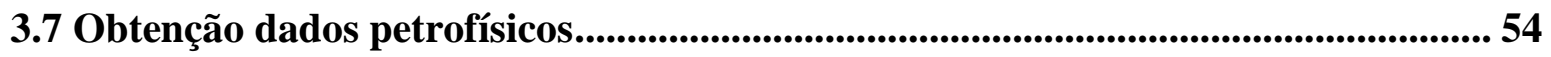

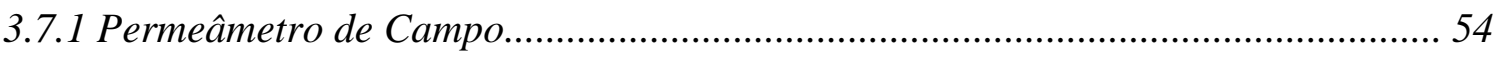

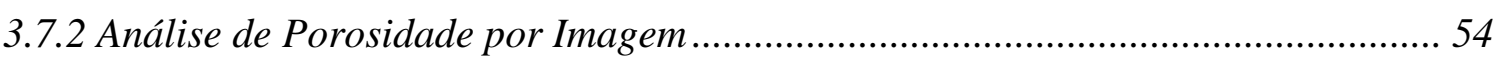

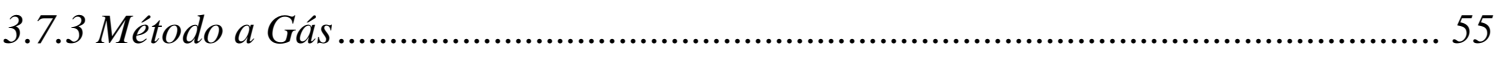

3.8 Calibração da Permeabilidade.......................................................................................... 57

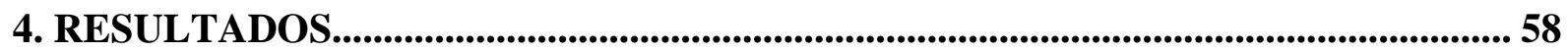

4.1 Caracterização Faciológica, Textural e Petrofísica da Formação Marizal ............. 58

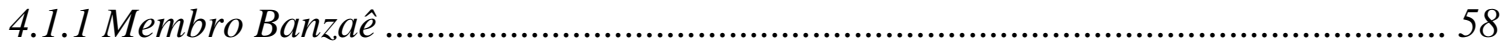

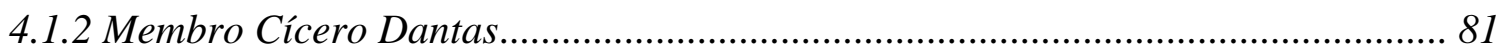

4.2 Análise de Proveniência e Diagênese da Formação Marizal ................................... 118

4.2.1 Membro Banzâ

4.2.1.1 Sistema Fluvial Transversal do Membro Banzaê ....................................... 119

4.2.1.2 Sistema Fluvial Axial do Membro Banzaê ................................................ 122

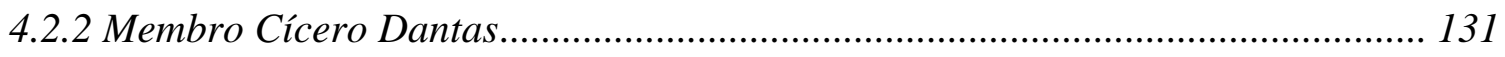

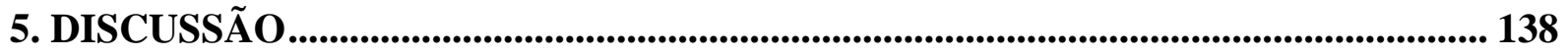

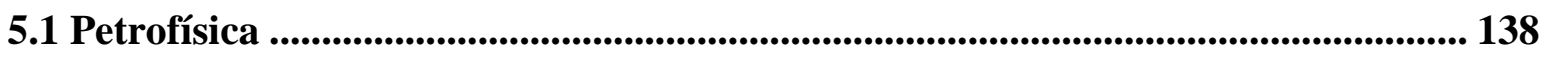

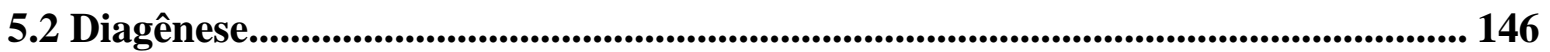

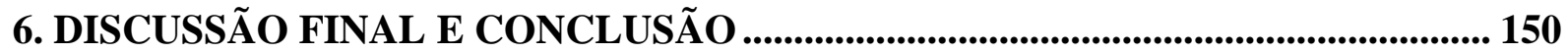

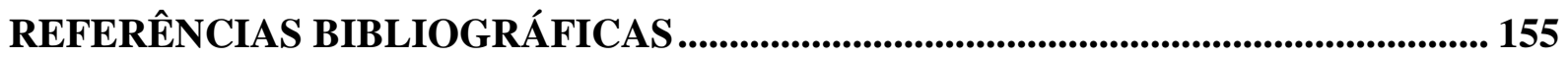




\section{INTRODUÇÃO}

O estudo de análogos de reservatórios é peça fundamental nas várias fases da indústria petrolífera seja na exploratória seja na de produção. Os análogos de afloramento vêm para suprir lacunas de resolução e volume de dados na indústria do hidrocarboneto, considerando que a sísmica apresenta baixa resolução vertical, enquanto o poço baixa resolução lateral (Alexander, 1993; Howell et al., 2014). Neste contexto diversos são os trabalhos que consideram a importância de estudos de afloramentos para o melhor entendimento da geometria, dimensões, distribuição das heterogeneidades sedimentares e potencial de conectividade entre os corpos, fatores que impactam no fluxo de hidrocarbonetos no reservatório (eg. Alexander, 1993; Hornung \& Aigner, 1999; Grammer et al., 2004; Noad, 2004; Larue \& Hovadik, 2006; Meyer \& Krauser, 2006; McKinley et al., 2011; Jackson et al., 2014; Howell et al., 2014; Keogh et al., 2014; Nordahl et al., 2014; Pranter et al., 2014).

O presente trabalho busca agregar novos conhecimentos para os estudos de análogos de reservatório em afloramentos com enfoque nas heterogeneidades em multiescala em sistemas fluviais através da caracterização faciológica, petrofísica e diagenética. Desta forma a região de estudo compreendeu a Formação Marizal (Aptiano, Bacia do Tucano, BA) que apresenta ótimas exposições de arenitos fluviais, recentemente estudadas por Freitas (2014), Carrera (2015) e Figueiredo et al. (2015).

Os afloramentos estudados estão inseridos no contexto do rifte abortado resultante da ruptura continental que originou o Oceano Atlântico Sul, durante o Eocretáceo (Magnavita et al., 2003; Magnavita et al. 2005). O rifte conhecido como Recôncavo-Tucano-Jatobá (RTJ) foi muito estudado por diversos autores (eg. Milani \& Davison, 1988; Magnavita, 1992; Lima \& Vilas Boas, 1994, 2000; Magnavita et al., 2003; Magnavita et al. 2005; Costa et al., 2007; Santos et al., 2010; Santos \& Reis, 2011), principalmente a Bacia do Recôncavo, considerando que foi a primeira bacia produtora do Brasil.

Para este trabalho foram estudados 8 afloramentos e um testemunho (de $100 \mathrm{~m}$ de comprimento) da Formação Marizal, recentemente subdivididos em membros Banzaê (basal) e Cícero Dantas por Freitas (2014) (Figura 1). Segundo este autor, o Membro Banzaê é composto por: (i) corpos de arenitos de sistemas fluviais interpretados como sucessão de cinturão de canal axial, denominados no presente trabalho como TUC-63 e SM e, na borda leste da bacia do Tucano Central, (ii) depósitos de leque aluvial e sistema fluvial transversal com paleocorrente para W-SW (afloramentos MR-03, MR-05 e NAT-01); por sua vez o Membro Cícero Dantas foi interpretado pelo autor como sendo composto por arenitos de um sistema fluvial de canais 
desconectados (afloramentos NAT-02, NAT-03, NAT-04 e SM) e por campos de dunas eólicas incipientes (afloramentos SI-34 e NAT-03).

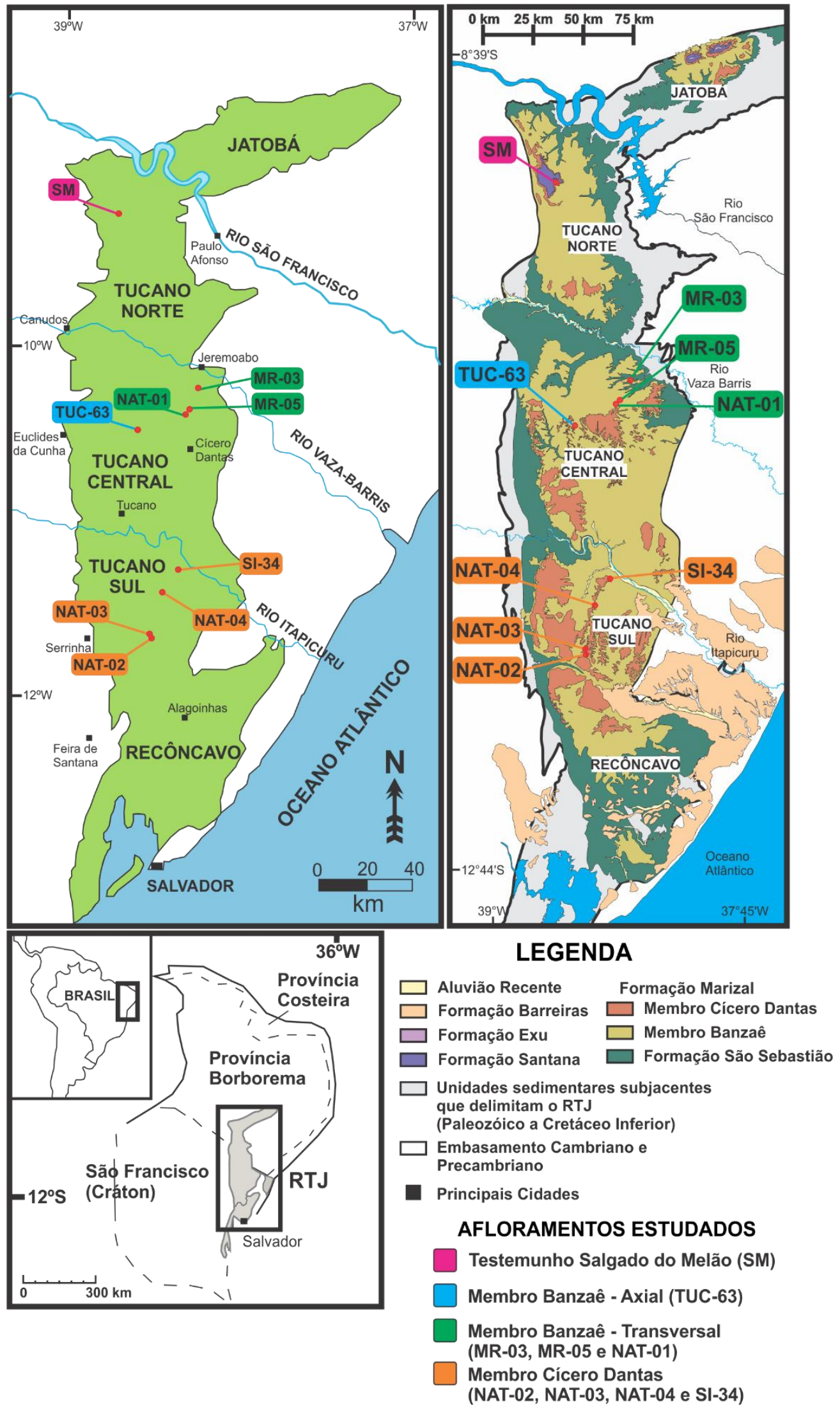

Figura 1. Mapa com localização geográfica dos afloramentos com principais cidades (esquerda) e mapa geológico com enfoque na distribuição da unidade Aptiana (extraído de Freitas, 2014) e também localização dos afloramentos em contexto geológico. 


\section{DISCUSSÃO FINAL E CONCLUSÃO}

O presente trabalho trouxe novos conhecimentos para caracterização de reservatórios fluviais em bacias distensionais. As pesquisas compreenderam estudos de fácies, elementos arquiteturais, diagenéticos e petrofísicos realizados nos arenitos da Formação Marizal (Bacia do Tucano, BA), sendo possível observar íntima relação entre os parâmetros geológicos e petrofísicos na caracterização de reservatórios.

Os processos diagenéticos identificados nos arenitos da Formação Marizal mostraramse diretamente relacionados com a proveniência do embasamento adjacente aos depósitos. Os arenitos interpretados por Freitas (2014), como depósitos fluviais transversais, apresentam paleocorrentes para oeste e para leste (testemunho SM - Membro Cícero Dantas) (Figura 104), com fragmentos líticos provenientes da Faixa Sergipana Subdomínio Macururé e Terreno Pernambuco-Alagoas, respectivamente (Figura 2). Esses depósitos foram classificados, de acordo com Folk (1974), como litoarenitos feldspáticos (MR-05 e MR-03) e arcóseos líticos (testemunho SM). Esses arenitos, com maiores quantidades de material lítico, sofreram processos mais intensos de compactação mecânica, o que afetou diretamente os valores de porosidade e permeabilidade (Figura 105A e B), também observado quando analisado isoladamente por fácies sedimentares (Figura 105C e D).

Os arenitos interpretados por Freitas (2014) como gerados por depósitos fluviais axiais, com paleocorrente para sul (afloramentos TUC-63, SI-34, NAT-02, NAT-03 e NAT-04) apresentam frações muito menores de fragmentos líticos, sendo classificados no presente trabalho segundo Folk (1974) como arcóseos (Figura 104).

Na porção norte da bacia, nota-se o controle que o embasamento teve na cimentação dos arenitos daquela localidade durante toda a sedimentação da bacia, conforme observado na caracterização diagenética do testemunho (SM), uma vez que a presença de rochas carbonáticas do Terreno Pernambuco-Alagoas (Figura 2) serviu como fonte para a precipitação do cimento carbonático que se formou nesses depósitos; assim como a presença de intraclastos lamosos em combinação com fluidos ricos em cálcio favoreceu a precipitação da dolomita.

Os arenitos do Membro Cícero Dantas, por apresentarem maior variabilidade de fácies de granulação fina (H e Alc) em depósitos de interduna e planície de inundação (com elevada quantidade de biotita), apresentam menores valores de permeabilidade em relação ao Membro Banzaê Axial (Figura 98A). Além disso, os arenitos do Membro Cícero Dantas apresentam elementos de barra que sofreram intenso processo telodiagenético, apresentando valores de permeabilidade semelhantes aos do Membro Banzaê Transversal (Figura 99A), esses depósitos do Membro Cícero Dantas apresentam intensa ferruginização (média, 5,8\%; máx., 19\%) e 
infiltração de argila (média, 4,5\%; máx., 14\%), destacando que a ferruginização é decorrente do intemperismo dos minerais de biotita presentes nesses depósitos de granulação mais fina no Membro Cícero Dantas.

Com isso, observa-se uma forte influência de diversos componentes na geração e qualidade de um reservatório, considerando desde da área-fonte, intemperismo, ambiente deposicional, compactação e diagênese para a qualidade dos reservatórios, o que implicará nas distintas características diagenéticas dos depósitos sedimentares. Em complemento, o presente trabalho demonstrou a importância de identificar os diversos tipos de depósitos dentro de uma bacia sedimentar, como nesse caso, onde se diferenciou uma história diagenética e compactacional diferente para os depósitos fluviais transversais e axiais.

Dessa forma, esses parâmetros devem ser levados em conta quando se trabalha com estudos de reservatórios independentemente do tipo de depósito e do tipo da bacia, pois em cada posição da bacia ocorrerão reservatórios com características distintas.

Uma importante contribuição do presente trabalho foram os dados diagenéticos analisados que indicaram para uma nova proposta para a caracterização do paleoclima da região, uma vez que a presença na eodiagênese de sobrecrescimento de k-feldspato, carbonatos e franjas de esmectita que evoluiram para interestratificados de I/S na mesodiagênese contribuem para a identificação de um ambiente semi-árido para o Aptiano, corroborando a proposta preliminar de Freitas (2014), em detrimento aos estudos de Chaboureau et al. (2003) que atribuíram um clima úmido para a região. 


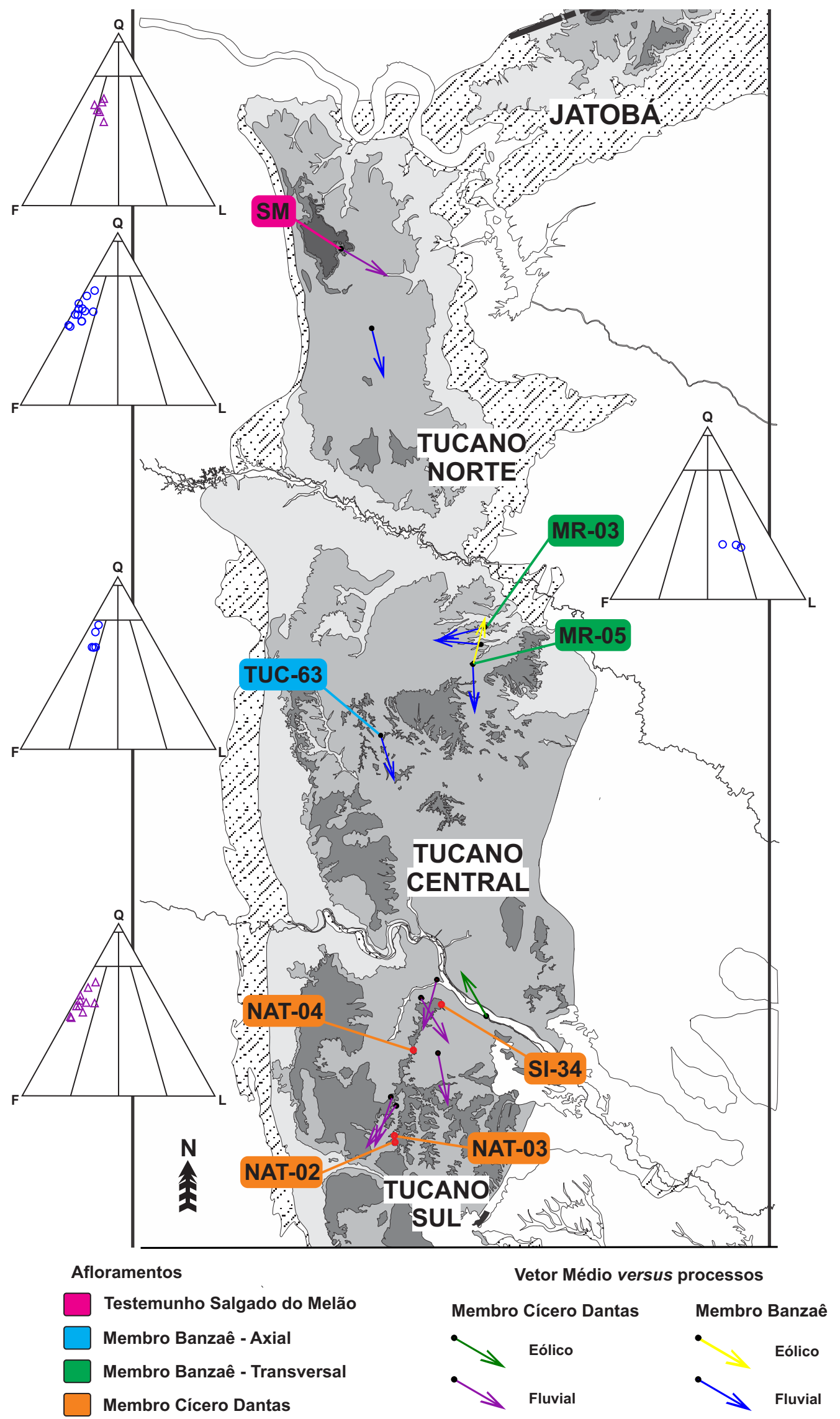

Figura 104. Mapa do rifte RTJ, com destaque para as sub-bacias Tucano, Sul, Central e Norte, localização dos afloramentos estudados, vetor médio da paleocorrente e gráficos QFL. As cores roxas nos gráficos QFL representam amostras do Membro Cícero Dantas, enquanto as azuis os do Membro Banzaê. Notar que vetores médios de paleocorrente transversais apresentam maior quantidade de fragmentos líticos e paleocorrentes axiais não apresentam essa distinção. Dados de paleocorrente foram compilados de Freitas (2014) e mapa modificado de Freitas (2014). 

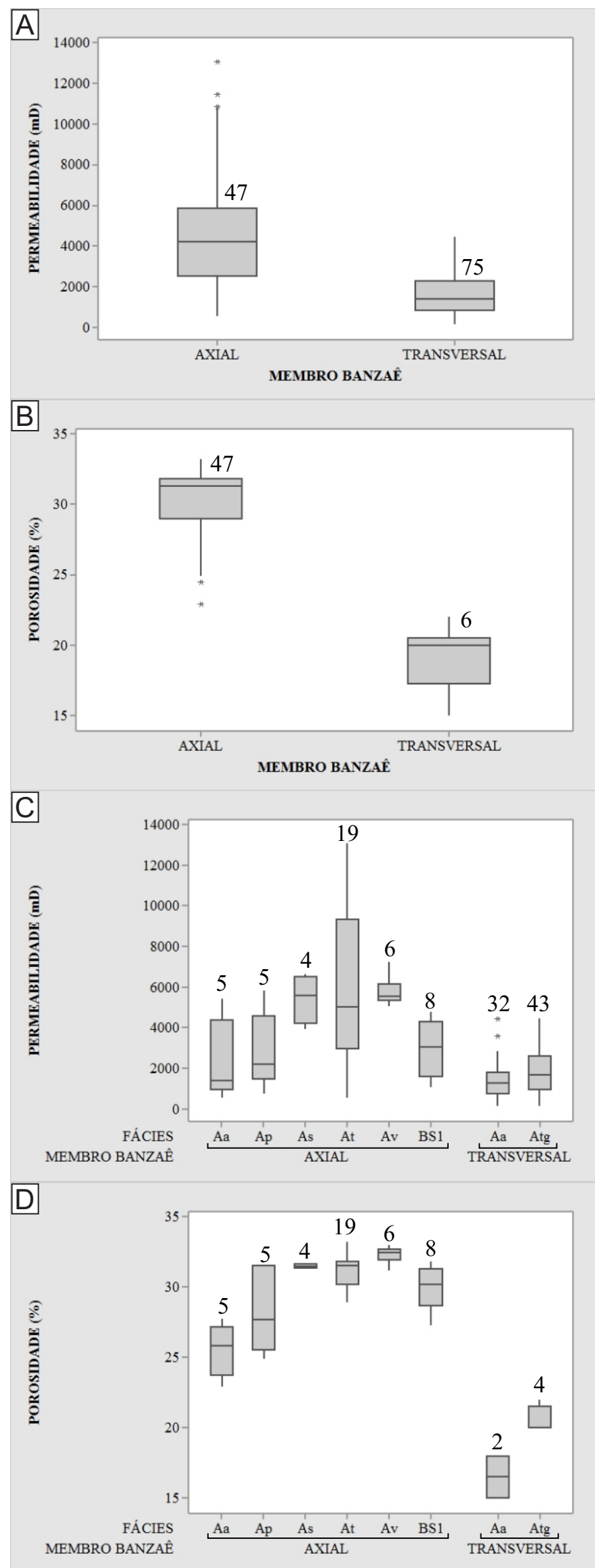

$\mathbf{A a}$ - arenito com estratificação cruzada acanalada Ap - arenito com estratificação cruzada de baixo ângulo ou plano-paralela As - arenito com estratificação cruzada sigmoidal At - arenito com estratificação cruzada tabular Av - cruzada de overturned BS1 - superfície limitante dos estratos cruzados compostos. Números representam quantidade de amostras.

Figura 105. Gráficos boxplot apresentam valores de porosidade e permeabilidade para os Membro Banzaê axial e transversal (A e B) e paras os mesmos membros em relação a fácies (C e D). Notar que mesma fácies presentes nos dois membros, fácies Aa, no membro transversal os valores de permeabilidade e porosidade são menores. 


\section{REFERÊNCIAS BIBLIOGRÁFICAS}

Ahlbrandt, T.S. \& Fryberger, S.G. 1981. Sedimentary features and significance of interdunes deposits. In: Ethridge, F.G. \& Flore, R.M. (eds), Recent and Ancient Non-marine Depositional Environments: Models for Explotation, SEPM Special Publication, 31: 293314.

Al-Masrahy, M.A. \& Mountney, N.P. 2015. A classification scheme for fluvial-aeolian system interaction in desert-margin settings. Aeolian Research, 17: 67-88.

Alexander, J. 1993. A discussion on the use of analogues for reservoir geology. In: Ashton, M. (ed), Advances in Reservoir Geology, Geological Society Special Publication, 69, 175-194.

Ali, S.A. 1981. Sandstone Diagenesis. Applications to Hydrocarbon Exploration and Production. Pittsburgh. Gulf Science \& Technology. 221 p.

Allen, J.R.L. 1963. The classification of cross-stratified units. With notes on their origin. Sedimentology, 2(3): 93-114.

Allen, J.R.L. 1983. Studies in fluvialtile sedimentation: bars, bar complexes and sandstone sheets (low-sinuosity braided streams) in the Brownstones (L. Devonian), Welsh Borders. Sedimentary Geology, 33: 237-293.

Almeida, F.F.M., Hasui, Y., Brito-Neves B.B., Fuck R.A. 1977. Províncias Estruturais Brasileiras. In: Simpósio de Geologia do Nordeste. Atas. Campina Grande. 363-391.

Almeida, F.F.M. Hasui, Y., Brito-Neves B.B., Fuck R.A. 1981. Brazilian Structural Provinces: an introduction. Earth Science Review, 17: 1-29.

Almeida, R.P., Freitas, B.T., Turra, B.B., Figueiredo, F.T., Marconato, A., Janikian, L. 2016. Reconstructing fluvial bar surfaces from compound cross-strata and the interpretation of bar accretion direction in large river deposits. Sedimentology, 63(3): 609-628.

Amaral, C.R.L. \& Brito, P.M., 2012. A new Chanidae (Ostariophysii: Gonorynchiformes) from the Cretaceous of Brazil with affinities to Laurasian Gonorynchiforms from Spain. PLoS ONE, 7 (5), e37247. doi:10.1371/journal.pone.0037247.

Alvarado-Ortega, J. \& Brito, P.M., 2010. A new ichthyodectiform (Actinopterygii, Teleostei) from the Lower Cretaceous Marizal Formation, northeast Brazil. Palaeontology, 53: 297306.

Best, J.L., Ashworth, P.J., Bristow, C.S., Roden, J. 2003. Three-dimensional sedimentary architecture of a large, mid-channel sand braid bar, Jamuna River, Bangladesh. Journal of Sedimentary Research, 73(4): 516-530.

Boggs, S.Jr. 1995. Principles of sedimentology and stratigraphy. Prentice-Hall, 2 edition, 774p. 
Bouza, P.J., Simón, M., Aguillar, J., del Valle, H., Rostagno, M. 2007. Fibrous-clay mineral formation and soil evolution in Aridisols of northeastern Patagonia, Argentina. Geoderma, 139: $38-50$.

BRASIL, 1948. Relatório de 1947, Estado da Bahia. Conselho Nacional do Petróleo. Rio de Janeiro, 91-182.

Bridge, J.S. 1993. Description and interpretation of fluvial deposits: a critical perspective. Sedimentology, 40: 801-810.

Bridge, J.S. 2003. Rivers and floodplains: forms, processes, and sedimentary record. Blackwell, $491 \mathrm{p}$.

Bridge, J.S. 2006. Fluvial facies models: recente developments. In: Posamentier, H.W. \& Walker, R.G. (eds), Facies Models Revisited, SEPM Special Publications, 84: 85-170.

Bridge, J.S. \& Demicco, R.V. 2008. Earth surface processes, landforms and sediment deposits. Cambridge University Press, New York, 815 p.

Bristow, C.S. 1993. Sedimentary structures exposed in bar tops in the Brahmaputra River, Bangladesh. In: Best, J.J \& Bristow, C.S. (eds), Braided Rivers, Geological Society, Special Publication, 75: 277-289.

Brito, P.M. \& Alvarado-Ortega, J., 2008. A new species of Placidichthys (Halecomorphi: Ionoscopiformes) from the Lower Cretaceous Marizal Formation, northeastern Brazil, with a review of the biogeographical distribution of the Ophiopsidae. In: Cavin, L., Longbottom, A., Richter, M. (Eds.), Fishes and the break-up of Pangea, Geological Society of London Special Publication, 295: 145-154.

Bull, W.B. 1977. The alluvial-fan environment. Progress in Physical Geography, 1:222-270.

Carrera, S.C. 2015. Arquitetura deposicional e proveniência da Formação Marizal nas subbacias Tucano Sul e Central, BA. Dissertação de Mestrado, Universidade de São Paulo, Brasil, 147 p.

Chaboureau, A.C., Guillocheau, F., Robin, C., Rohais, S., Moulin, M., Aslanian. 2013. Paleogeographic evolution of the central segment of the South Atlantic during Early Cretaceous times: Paleotopographic and geodynamic implications. Tectonophysics, 604: 191-223.

Chan, M.A. \& Kocurek, G. 1988. Complexities in eolian and marine interactions - processes and eustatic controls on erg development. Sedimentary Geology, 56: 283-300.

Chandler, M.A., Kocurek, G., Goggin, D.J., Lake, L.W. 1989. Effects of stratigraphic heterogeneity on permeability in eolian sandstone sequence, Page Sandstone, northern Arizona. AAPG Bulletin, 73: 658-668. 
Choquette, P.W. \& Pray, L.C.L. 1970. Geologic nomenclature and classification of porosity in sedimentary carbonates. Am. Assoc. Petrol. Geol. Bulletin 54: 207-250.

Costa, I.P., Milhomem, P.S., Bueno, G.V., Silva, H.S.R.L., Kosin, M.D. 2007. Sub-bacias de Tucano Sul e Central. Boletim de Geociências da Petrobrás, 15(2): 433-443.

Cruz, D.A. 2011. Calibração e verificação das medidas de um permeâmetro. Dissertação de mestrado, Universidade Petrobrás, 55p.

De Ros, L.F. 1996. Compositional controls in sandstone diagenesis. Acta Universitatis Upsaliensis, Comprehensive Summaries of Uppsala Dissertations from the Faculty of Science and Technology 198. 24 p. Uppsala.

De Ros, L.F., Goldberge, K., Abel, M., Victorinetti, F., Mastella, L., Castro, E. 2007. Advanced acquisition and management of petrographic information from reservoir rocks using the Petroledge ${ }^{\circledR}$ System. AAPG Conference and Exhibition, Long Beach, CA, USA, Extended Abstract Vol. 6p.

De Ros, L.F. \& Scherer, C.M.S. 2012. Stratigraphic controls on the distribution of diagenetic processes quality and heterogeneity of fluvial-aeolian reservoirs from the Recôncavo Basin, Brazil. Int. Assoc. Sedimentol. Spec. Publ. 45: 105-132.

Delgado, I.M., Souza, J.D., Silva, L.C., Silveira Filho, N.C., Santos, R.A., Pedreira, A.J., Guimarães, J.T., Angelim, L.A.A., Vasconcelos, A.M., Gomes, I.P., Lacerda Filho, J.V., Valente, C.R., Perrotta, M.M., Heinec, C.A. 2003. Geotectônica do Escudo Atlântico. In: Bizzi, L.A., Schobbenhaus, C., Vidotti, R.M., Golçalves, J.H. (eds). Geologia, tectônica e recursos minerais do Brasil. Brasília. Companhia de Recursos Minerais, 227-334.

Deschamps, R., Kohler, E., Gasparrini, M., Durand, O., Euzen, T., Nader, F. 2012. Impact of mineralogy and diagenesis on reservoir quality of the Lower Cretaceous Upper Mannville Formation (Alberta, Canada). Oil \& Gas Science and Technology 67(1): 31-58.

Dutton, S.P. \& Land, L.S. 1985. Meteorical burial diagenesis of Pennsylvanian arkosic sandstones, southwestern Anadarko Basin, Texas. AAPG Bulletin, 69: 22-38.

Figueiredo, F.T. 2013. Proveniência e arquitetura de depósitos fluviais das Sub-bacias Tucano Central e Norte, Cretáceo (BA). Tese de Doutorado, Universidade de São Paulo, Brasil, $193 \mathrm{p}$.

Figueiredo, F.T., Almeida, R.P., Freitas, B.T., Marconato, A., Carrera, S.C., Turra, B.B. 2015. Tectonic activation, source area stratigraphy and provenance changes in a rift basin: the Early Cretaceous Tucano Brasin (NE-Brazil). Basin Research. doi:10.1111/bre.12115.

Freitas, B.T. 2014. A Formação Marizal (Aptiano) na Bacia do Tucano (BA): contribuições à análise da arquitetura de depósitos fluviais e implicações paleobiogeográficas. Tese de Doutorado, Universidade de São Paulo, Brasil, 175 p. 
Folk, R.L. 1974. Petrology of sedimentary rocks. Hemphill, Austin, Texas, 182 p.

Gaupp, R., Matter, A., Platt, J., Ramseyer, K., Walzebuck, J. 1993. Diagenesis and fluid evolution of deeply buried Permian (Rotliegend) gas reservoirs, northwest Germany. AAPG Bulletin, 77: 1111-1128.

Galeazzi, C. P. 2012. Avaliação de influência da zona de transferência do vaza-barris na deposição das Formações São Sebastião (Barremiano) e Marizal (Aptiano), Bacia do Tucano (BA). Monografia de Trabalho de Formatura, Instituto de Geociências, Universidade de São Paulo, 46 p.

Gava, A., Nascimento, D.A., Vidal, J.L.B., Ghignone, J.I.P., Santiago Filho, A.L., Teixeira, W., Stanford, W.J.P., Ribeiro, A.G., and Ribeiro, J.H.M., 1983. Geologia. In: Projeto RADAMBRASIL Folha SC.24/25 Aracaju/Recife. Rio de Janeiro, MME/SG. p. 27-376 (Levantamento de Recursos Naturais, 30).

Gesicki, A.L.D., Sayeg, I.J., Curti, D.K., Boggiani, P.C., Giannini, P.C.F. 2009. Determinação quantitativa de parâmetros texturais de arenitos eólicos através de análise de imagens digitais de seções delgadas. Revista Brasileira de Geociências, 39(2): 267-275.

Goudie, A.S. 1983. Calcrete. In: Goudie, A.S \& Pye, K. (eds), Chemical Sediments and Geomorphology: Precipitates and Residues in the Near-surface Environment, Academic Press, San Diego, 93-131.

Grammer, G.M., Harris, P.M., Eberli, G.P. 2004. Integration of outcrop and modern analogs in reservoir modeling: overview with examples from the Bahamas. AAPG Memoir, 80: 1-20.

Guimarães, J.T. Projeto Bacia do Tucano Sul: Estado da Bahia. Salvador: CPRM, 2002. 55 p. il. Escala 1:200.000. Programa Levantamentos Geológicos Básicos do Brasil.

Hall, J.S., Mozley, P., Davis, J.M., Roy, N.D. 2004. Environments of formation and controls on spatial distribution of calcite cementation in Plio-Pleistocene fluvial deposits, New Mexico, USA. Journal of Sedimentary Research 74(5): 643-653.

Henares, S., Caracciolo, L., Cultrone, G., Fernández, J., Viseras, C. 2014. The role of diagenesis and depositional facies on pore system evolution in a Triassic outcrop analogue (SE Spain). Marine and Petroleum Geology 51: 136-151.

Hornung, J. \& Aigner, T. 1999. Reservoir and aquifer characterization of fluvial architectutal elements: Stubensandstein, Upper Triassic, southwest Germany. Sedimentary Geology, 129: 215-280.

Houseknecht, D.W. 1987. Assessing the relative importance of compaction processes and cementation to reduction of porosity in sandstones. AAPG Bulletin, 71(6): 633-642.

Howell, J.A., Martinius, A.W., Good, T.R. 2014. The application of outcrop in geological modelling: a review, present status and future outlook. In: Matinius, A.W., Howell, J.A., 158 
Good, T.R. (eds), Sediment-Body Geometry and Heterogeneity: Analogue Studies for Modelling the Subsurface, Geological Society, London, Special Publications, 387: 1-25.

Howard, J.J. 1981. Lithium and potassium saturation of illite/smectite clays from interlaminated shales and sandstones. Clays and Clays Minerals, 29(2): 136-142.

Hunter, R.E. 1977. Basic types of stratification in small eolian dunes. Sedimentology, 24: 361387.

Jackson, M.D., Hampson, G.J., Saunders, J.H., El-Sheikh, A., Graham, G.H., Massart, B.Y.G. 2014. Surface-based reservoir modelling for flow simulation. In: Martinius, A.W., Howell, J.A., Good, T.R. (eds), Sediment-Body Geometry and Heterogeneity: Analogue Studies for Modelling the Subsurface, Geological Society, London, Special Publication, 387.

Jonas, E.O. \& McBride, E.F. 1977. Diagenesis of sandstone and shale: Application to exploration for hydrocarbons. Cont. Educat. Program. 120p

Keogh, K.J., Leary, S., Martinius, A.W., Scott, A.S.J., Riordan, S., Viste, I., Gowland, S., Taylor, A.M., Howell, J. 2014. Data capture for multiscale modelling of the Lourinhã Formation, Lusitana Basin, Portugal: an outcrop analogue for the Statfjord Group, Norwegian North Sea. In: Martinius, A.W., Howell, J.A., Good, T.R. (eds), Sediment-Body Geometry and Heterogeneity: Analogue Studies for Modelling the Subsurface, Geological Society, London, Special Publication, 387.

Khidir, A. \& Catuneanu, O. 2003. Sedimentology and diagenesis of the Scollard sandstones in the Red Deer Valley area, central Alberta. Bulletin of Canadian Petroleum Geology 51(1): 45-69.

Khidir, A. \& Catuneanu, O. 2009. Basin-scale distribution of authigenic clay minerals in the Late Maastrichtian-Early Paleocene fluvial strata of the Alberta foredeep: implications for burial depth. Bulletin of Canadian Petroleum Geology 57(3): 251-274.

Khidir, A. \& Catuneanu, O. 2010. Diagenesis of the Cretaceous-Tertiary Willow Creek sandstones, southwestern region of Alberta. Bulletin of Canadian Petroleum Geology 58(4): 342-360.

Kocurek, G. 1981. Significance of interdune deposits and bounding surfaces in Aeolian dune sands. Sedimentology, 28: 753-780.

Kocurek, G. 1988. First-order and super bounding surfaces in eolian sequences - Bounding surfaces revisited. Sedimentary Geology, 56: 193-206.

Kocurek, G. 1991. Interpretation of ancient eolian sand dunes. Annual Review of Earth and Planetary Sciences, 19: 43-75.

Kocurek, G. 1996. Desert Aeolian systems. In: Reading, H.C. (ed), Sedimentary Environments: Process, Facies and Stratigraphy, Blackwell Science, 125-153. 
Kocurek, G. \& Nielson, J. 1986. Conditions favourable for the formation of warm-climate aeolian sand sheets. Sedimentology, 33: 795-816.

Kocurek, G., Robinson, N.I., Sharp, J.M.Jr. 2001. The response of the water table in coastal aeolian systems to changes in sea level. Sedimentary Geology, 139: 1-13.

Leopold, L.B. \& Wolman, M.G. 1957. River patterns, braided, meandering and straight. U.S. Geological Survey Profissional Paper, 282-B: 1-85.

Larue, D.K. \& Hovadik, J. 2006. Connectivity of channelized reservoirs: a modelling approach. Petroleum Geoscience, 12: 291-308.

Lima, C. \& Vilas Boas, G., 1994. Mecanismos de transporte e deposição dos Conglomerados da Formação Marizal (Cretáceo Inferior) na Bacia do Recôncavo, Bahia, Brasil. Brazilian Journal of Geology, 24: 240-246.

Lima, C. \& Vilas Boas, G., 2000. A arquitetura deposicional da Formação Marizal (Cretáceo Inferior) na Bacia do recôncavo, Bahia. Brazilian Journal of Geology, 30(4): 729-736.

Lindquist, S.J. 1988. Practical characterization of eolian reservoirs for development: Nugget Sandstone, Utah - Wyoming thrust belt. Sedimentary Geology, 56: 315-339.

Luo, J.L., Morad, S., Salem, A., Ketzer, J.M., Yan, S., Zhang, X.L., Xue, J.M., Hlal, O. 2009. Impact of diagenesis on reservoir-quality evolution in fluvial and lacustrine-deltaic sandstones: evidence from Jurassic and Triassic sandstones from the Ordos basin, China. Journal of Petroleum Geology 32(1): 79-102.

Magnavita, L.P. \& Cupertino, J.A., 1987. Concepção atual sobre as bacias do Tucano e Jatobá, Nordeste do Brasil. Boletim de Geociências da Petrobras, 1: 119-134.

Magnavita, L.P., 1992. Geometry and kinematics of the Recôncavo-Tucano-Jatobá Rift, NE Brazil: Ph.D. thesis, University of Oxford, England.

Magnavita, L.P., Davison, I., Kusznir, N.J., 1994. Rifting, erosion, and uplift history of the Recôncavo-Tucano-Jatobá Rift, northeast Brazil. Tectonics, 13: 367-388.

Magnavita, L.P., Destro, N., Carvalho, M.S.S., Milhomem, P.S., Souza-Lima, W., 2003.

Bacia de Tucano. Aracaju, Fundação Paleontológica Phoenix, Séries Bacias Sedimentares, ano $5, \mathrm{n} 52$.

Magnavita, L.P., Silva, R.S., Sanches, C.P., 2005. Roteiros geológicos, guia de campo da Bacia do Recôncavo, NE do Brasil. Boletim de Geociências da Petrobrás, 13: 301-334.

Maraschin, A.J., Mizusaki, A.M.P., De Ros, L.F. 2004. Near-surface k-feldspar precipitation in Cretaceous sandstones from the Potiguar Basin, Northeastern Brazil. The Journal of Geology 112: 317-334. 
McBride, E.F., Land, L.S., Mack, L.E. 1987. Diagenesis of eolian and fluvial feldspathic sandstones, Norphlet Formation (Upper, Jurassic), Rankin County, Mississippi, and Mobile County, Alabama. AAPG Bulletin, 71: 1019-1034.

McKinley, J.M., Worden, R.H., Ruffell, A.H. 2003. Smectite in sandstones: a review of the controls on occurrence and behavior during diagenesis. In: Worden, R.H. \& Morad, S. (eds), Clay Mineral Cements in Sandstones, Int. Assoc. Sedimentol. Spec. Publ., 34: 109128.

McKinley, J.M., Atkinson, P.M., Lloyd, C.D. Ruffell, A.H., Worden, R.H. 2011. How porosity and permeability vary spatially with grain size, sorting, cement volume, and mineral dissolution in Fluvial Triassic Sandstone: the value of geostatistic and local regression. Journal of Sedimentary Research, 81: 844-858.

Meyer, R. \& Krause, F.F. 2006. Permeability anisotropy and heterogeneity of a sandstone reservoir analogue: an estuarine to shore depositional system in the Virgelle Member, Milk River Formation, Writing-on-Stone Provincial Park, southern Alberta. Bulletin of Canadian Petroleum Geology, 54(4): 301-318.

Miall, A.D. 1977. A review of the braided-river depositional environment. Earth Science Reviews, 13: 1-62.

Miall, A.D. 1985. Architectural-element analysis: a new method of facies analysis applied to fluvial deposits. Earth Science Reviews, 22: 261-308.

Miall, A.D. 1996. The geology of fluvial deposits. Springer, $582 \mathrm{p}$.

Miall, A.D. 2000. Principles of sedimentary basin analysis. Springer-Verlag (3 ed.), 616 p.

Milani, E.J. \& Davison, I., 1988. Basement control and transfer tectonics in the RecôncavoTucano-Jatobá rift, Northeast Brazil. Tectonophysics, 154: 41-70.

Morad, S. 1998. Carbonate cementation in sandstones: distribution patterns and geochemical evolution. In: Morad, S. (ed), Carbonate Cementation in Sandstones, IAS Special Publication, 26: 1-26.

Morad, S., De Ros, L.F., Nystuen, J.P., Bergan, M. 1998. Carbonate diagenesis and porosity evolution in sheet-flood sandstones: evidence from the Middle and Lower Lunde Members (Triassic) in the Snorre Field, Norwegian North Sea. In: Morad, S. (ed), Carbonate Cementation in Sandstones, IAS Special Publication, 26: 53-85.

Morad, S., Ketzer, J.M., De Ros, L.F. 2000. Spatial and temporal distribution of diagenetic alterations in siliciclastic rocks: implications for mass transfer in sedimentar basins. Sedimentology 47: 95-120. 
Morad, S; Al-Ramadan, K; Ketzer, JM; De Ros, LF. 2010. The impact of diagenesis on the heterogeneity of sandstone reservoirs: A review of the role of deposicional facies and sequence stratigraphy. AAPG Bulletin, 94(8): 1267-1309.

Moraes, M.A.S. \& De Ros, L.F. 1990. Infiltrated clays in fluvial Jurassic sandstones of Recôncavo Basin, northeastern Brazil. Journal of Sedimentary Petrology, 60: 809-819.

Moraes, M.A.S \& De Ros, L.F. 1992. Depositional, infiltrated and authigenic clays in fluvial sandstones of the Jurrassic Sergi Formation, Recôncavo Basin, northeastern Brazil. In: Origin, diagenesis and petrophysics of clay minerals in sandstones, Houseknecht, D.W., Pittman, E.W. (eds). SEPM Special Publication 47: 197-208.

Mota, F.O.B., Oliveira, J.B., Gebhardt, H. 2002. Mineralogia de um argissolo vermelhoamarelo eutrófico e de um planossolo háplico eutrófico solódico numa topossequência de gnaisse no sertão central do Ceará. R. Bras. Ci. Solo, 26: 607-618.

Mountney, N.P. 2006. Eolian facies models. In: Posamentier, H.W. \& Walker, R.G. (eds), Facies Models Revisited, SEPM Special Publications, 84: 19-83.

Nichols, G. 2009. Sedimentology and Stratigraphy. Wiley-Blackwell (2 ed.), 419p.

Noad, J. 2004. The use of field analogues in the correlation and static reservoir methodology used in the Tern Field, Nothern North Sea, UK. Marine and Petroleum Geology, 21: 485497.

Nordahl, J., Messina, C., Berland, H., Rustad, A.B., Rimstad, E. 2014. Impact of multiscale modelling on predicted porosity and permeability distributions in the fluvial deposits of the Upper Lunde Member (Snorre Field, Norwegian Continental Shelf). In: Martinius, A.W., Howell, J.A., Good, T.R. (eds), Sediment-Body Geometry and Heterogeneity: Analogue Studies for Modelling the Subsurface, Geological Society, London, Special Publication, 387.

Perry, E. \& Hower, J. 1970. Burial diagenesis in Gulf Coast politic sediments. Clays and Clay Minerals, 18: 165-177.

Pettijohn, F.L. 1957. Sedimentary Rocks. Ney York, Harper \& Row Publishers, 719p.

Pittman, E.D. 1979. Porosity, diagenesis and productive capability of sandstone reservoirs. In: Scholle, P.A. \& Schluger, P.R. (eds), Aspects of Diagenesis, SEPM Special Publications, 26: $159-173$.

Pranter, M. J., Hewllet, A.C., Cole, R.D., Wang, H., Gilman, J. 2014. Fluvial architecture and connectivity of the Williams Fork Formation: use of outcrop analogues for stratigraphic characterization and reservoir modelling. In: Martinius, A.W., Howell, J.A., Good, T.R. (eds), Sediment-Body Geometry and Heterogeneity: Analogue Studies for Modelling the Subsurface, Geological Society, London, Special Publication, 387. 
Purvis, K. 1994. Extensive albite dissolution in Triassic reservoir sandstones from the Gannet field, UK North Sea. Marine and Petroleum Geology 11(5): 624-630.

Pye, K. 1982. Negatively skewed Aeolian sands from a humid tropical coastal dunefield, northern Australia. Sedimentary Geology, 31: 249-266.

Pye, K. \& Tsoar, H. 1990. Aeolian sand and sand dunes. London, Unwin, 369p.

Ramm, M. \& Ryseth, AE. 1996. Reservoir quality and burial diagenesis in the Statfjord Formatiuon, North Sea. Petroleum Geoscience 2: 313-324.

Reading, H.G. 1986. Facies. In: Reading, H.G. (ed.), Sedimentary Environments and Facies, $2^{\text {nd }}$ edition, Blackwell, 4-19.

Reading, H.G. 1996. Sedimentary Environments: Processes, Facies and Stratigraphy. Blackwell, 688 p.

Reed, J.S., Eriksson, K.A., Kowalewski, M. 2005. Climatic, depositional and burial controls on diagenesis of Appalachian Carboniferous sandstones: qualitative and quantitative methods. Sedimentary Geology 176: 225-246.

Reesink, A.J.H. \& Bridge, J.S. 2007. Influence of superimposed bedforms and flow unsteadiness on formation of cross strata in dunes and unit bars. Sedimentary Geology, 202: $281-296$.

Reesink, A.J.H. \& Bridge, J.S. 2009. Influence of superimposed bedforms and flow unsteadiness on formation of cross strata in dunes and unit bars - Part 2, further experiments. Sedimentary Geology, 222: 274-300.

Reesink, A.J.H., Ashworth, P.J., Sambrook Smith, G.H., Best, J.L., Parsons, D.R., Amsler, M.L., Szupiany, R.N. 2014. Scales and causes of heterogeneity in bars in a large multichannel river: Río Paraná, Argentina. Sedimentology, 61(4): 1055-1085.

Rezaee, M.R. \& Lemon, N.M. 1996. Influence of depositional environment on diagenesis and reservoir quality: Tirrawarra sandstone reservoir, southern Cooper Basin, Australia. Journal of Petroleum Geology 19(4): 369-391.

Rossi, C., Marfil, R., Ramseyer, K., Permanyer, A. 2001. Facies-related diagenesis and multiphase siderite cementation and dissolution in the reservoir sandstones of the Khatatba Formation, Egypt's Western Desert. Journal of Sedimentary Research 71(3): 459-472.

Salem, A.M., Morad, S., Mato, L.F., Al-Aasm, I.S. 2000. Diagenesis and reservoir-quality evolution of fluvial sandstones during progressive burial and uplift: evidence from the Upper Jurassic Boipeba Member, Recôncavo Basin, Northeastern Brazil. AAPG Bulletin 7: 1015-1040.

Sambrook Smith, G.H., Ashworth, P.J., Best, J.L., Woodward, J., Simpson, C.J., 2006. The 
sedimentology and alluvial architecture of the sandy braided South Saskatchewan River, Canada. Sedimentology, 53: 413-434.

Sambrook Smith, G.H., Ashworth, P.J., Best, J.L., Lunt, I.A., Orfeo, O., Parsons, D.R., 2009. The sedimentology and alluvial architecture of a large braid bar, Rio Paraná, Argentina. Journal of Sedimentary Research, 79(8): 629-642.

Santos, C.C., Reis, C., Pedreira, A.J., 2010. Projeto Bacia do Tucano Central: Folha Ribeira do Pombal - SC.24-Z-A-IV; Folha Cícero Dantas - SC.24-Z-A-V. Estado da Bahia e Sergipe. Salvador: CPRM, 2010. 87 p. il. Escala 1:100.000. Programa Geologia do Brasil. Levantamentos Geológicos Básicos.

Santos, C.C. \& Reis, C., 2011. Projeto Bacia do Tucano Central: Folha Caimbé - SC.24-Z-A-I, Folha Jeremoabo - SC.24-Z-A-II: Estado da Bahia. Salvador: CPRM, 2011. 99 p. il. Escala 1:100.000. Programa Geologia do Brasil. Levantamentos Geológicos Básicos.

Sawakuchi, A.O. 2006. Morfometria e simulação numérica de campus de dunas costeiros: estudo baseado em exemplos brasileiros. Tese de Doutorado, Universidade de São Paulo, Brasil, $316 \mathrm{p}$.

Scherer, C.M.S \& De Ros, L.F. 2009. Heterogeneidades dos reservatórios flúvio-eólicos da Formação Sergi na Bacia do Recôncavo. Bol. Geoc. Petrobras 17(2): 249-271.

Schmidt, V. \& McDonald, D. 1979. The role of secundary porosity in the course of sandstone diagenesis. In: Aspects of diagenesis, Scholle, P.A. \& Schluger, P.R. (eds). SEPM Special Publication 26: 175-207.

Schumm, S.A. 1985. Patterns of aluvial rivers. Annual Reviews of Earth and Planetary Science, 13: 5-27.

Seeman, U. 1982. Depositional facies, diagenetic minerals and reservoir quality of Rotliegende sediments in the southern Permian Basin (North Sea): A Review. Clay Minerals, 17: 5567.

Selley, R.C. 1998. Elements of Petroleum Geology. Academic Press, $2^{\text {nd }}$ edition, 470p.

Shepherd, M. 2009. Eolian reservoir. In: Sheperd, M. (ed), Oil field production geology, AAPG Memoir, 91: 255-259.

Silva Santos, R. 1972. Peixes da Formação Marizal, Estado da Bahia. Tese de Doutoramento, Universidade de São Paulo, Instituto de Geociências, São Paulo, 76 p.

Silva, C.M.A. \& Anjos, S.M.C. 1996. Diagênese dos arenitos do Membro Mucuri, Cretáceo Inferior das Bacias do Espírito Santo e de Mucuri. Boletim de Geociências Petrobrás 10(1/4): 61-80.

Strong, G.E. \& Milodowski, A.E. 1987. Aspects of the diagenesis of the Sherwood Sandstones of the Wessex Basin and their influence on reservoir characteristics. In: Marshall, JD (ed), 
Diagenesis of Sedimentary Sequences, Geological Society Special Publications 36: 325337.

Taylor, J.C.M. 1978. Control of diagenesis by depositional environment within a fluvial sandstone sequence in the northern North Sea Basin. Journal of the Geological Society 135: 83-91.

Tiab, D. \& Donaldson, E.C. 2004. Petrophysics theory and practice of measuring reservoir rock and fluid transport properties. Elsevier, $2^{\text {nd }}$ edition, 889p.

Varejão, F.G. 2016. Estratigrafia da Andar Alagoas na Sub-Bacia do Tucano Norte, Bahia. Dissertação de mestrado, Universidade Estadual Paulista, Brasil, 96 p.

Viana, C.F., Junior, E.G.G., Simões, I.A., Moura, J.A., Fonseca, J.D.R., Alves, R.J., 1971. Revisão estratigráfica da Bacia Recôncavo/Tucano. Boletim Técnico da Petrobrás, 14: 157-192.

Walker, T.R. 1976. Diagenetic origin of continental Red Beds. In: Falke, H (ed), The Continental Permian in Central, West and South Europe, D. Reidel Publishing Company, 240-282.

Walker, T.R., Waugh, B., Crone, A.J. 1978. Diagenesis in first-cycle desert alluvium of Cenozoic age, south-western United states and northwestern Mexico. Geological Society of America Bulletin 89: 19-32.

Walker, R.G. \& Cant, D.J. 1984. Sandy fluvial systems. In: Walker, R.G. (ed.), Facies Models (2 ed.), Geoscience Canada Reprint Series, 1: 71-89.

Wilson, M.J. 1999. The origin and formation of clay minerals in soils: past, present and future perspectives. Clay Minerals, 34: 7-25.

Worden, R.H. \& Burley, S.D. 2003. Sandstone diagenesis: the evolution of the sand to stone. In: Burley, S.D. \& Worden, R.H. (eds), Sandstone Diagenesis: Recent and Ancient, Reprint series volume 4 of the IAS, 4-44.

Worden, R.H. \& Morad, S. 2000. Quartz cementation in oil field sandstones: a review of the key controversies. In: Worden, R.H. \& Morad, S. (eds), Quartz Cementation in Sandstones, Int. Assoc. Sedimentol. Spec. Publ., 29: 1-20.

Worden, R.H. \& Morad, S. 2003. Clay minerals in sandstones: controls on formation, distribution and evolution. In: Worden, R.H. \& Morad, S. (eds), Clay Mineral Cements in Sandstones, Int. Assoc. Sedimentol. Spec. Publ., 34: 3-41.

Zuffa, G.G. 1985. Optical analysis of arenites: influence of methodology on compositional results. In: Zuffa, G.G (ed.), Provenance of Arenites, D. Reidel Publishing Company, 165189. 